recording materials of improved phase-recording quality could be found, then several uses could be foreseen for composite reflectors containing a very large number of stacked Lippman reflecting layers. He referred also to the increasing American interest in acoustic holography, which could be an attractive alternative to $\mathrm{X}$-rays in medical diagnosis.

Of the papers that followed, two dealt with ultrasonics and with the problems of recording particle tracks in a bubble chamber, and three were concerned with fundamental optical aspects of holography. The paper by J. W. C. Gates (NPL) emphasized the advantages of pulsed laser holography and included a live demonstration in which a hologram was handed round the audience only minutes after it had been recorded on the platform.

The remaining papers dealt with measuring applications and made it clear that, although holography as a means of processing information has been studied for many years, its more recent cole as a measuring tool is at least equally important.

The paper by A. E. Ennos (NPL) reviewed some of the ways in which hologram interferometry can be used to measure either minute changes in the shape of an engineering component or departures from a holographically recorded master shape. The highlight of this presentation was a short cine film which demonstrated the advantage of a new stroboscopic method, as opposed to the time averaged technique, for studying the vibrations of an acoustic resonator the resonances of which are sharp. J. M. Burch (NPL) described a new holographic version. of the "moiré shadow" test which should make it possible to test a diffusely reflecting component such as a turbine blade with a reduced sensitivity of perhaps 400 fringes per centimetre of error. W. R. B. Bradford (EMI) illustrated the experimental precautions that are needed for holographic metrology, and showed results that should be of forensic as well as of engineering interest. It appears, for example, that if a floorboard is dented, Dr Bradford can determine which if any of the dents is less than an hour old.

The brief symposium was accompanied by several holographic demonstrations which were arranged by the NPL Division of Optical Metrology in an adjoining laboratory. A high degree of interest was shown throughout, and this augurs well for a full international conference on "Engineering Uses of Holography" which is to be held at the University of Strathclyde between September 17 and 20 .

\section{Extraction by Bacteria}

\section{from our Microbiology Correspondent}

Fourteen years ago it was found that certain sulphuroxidizing bacteria could accelerate the extraction of copper sulphide ores. Considerable use is now made of this leaching process which has rapidly attained importance as the necessity to exploit low-grade ores has arisen. This extraction process has been studied both in the laboratory and the field, and reports in the current number of Biotechnology and Bioengineering indicate the present state of our knowledge and the problems involved in optimizing the conditions for a successful commercial operation. Beck (Biotech. Bioeng., IX, 487; 1967) has surveyed the practice of copper leaching in several mines in the western United
States and, although the control of the process and the extent of the recovery varied greatly, essentially the same proccdure was cmployed at all mines. Where convenient, the waste or low-grade ore (up to 20 million tons) is deposited in a ravine which forms a natural collection system and water is percolated through the ore dump. Pregnant waters rich in soluble copper and ferric iron feed a collection pond and then are piped to a precipitation plant where the copper is recovered and the iron is in part reduced to ferrous sulphate. Spent waters are subjected to further microbiological action in an oxidation pond in which iron is precipitated as hydrated ferric sulphate with a concomitant decrease in acidity. Finally, the spent waters are recycled through the ore dump and sulphuric acid added en route to maintain optimal $p \mathrm{H}$ conditions. Beck's survey proved a consistent association of Thiobacillus ferrooxidans with leaching waters and exposed ores and a massive development of this bacterium in the oxidation ponds. Stimulation of thiobacilli in this latter site provides a large inoculum in the percolate and removes much of the iron which might precipitate and cement in the upper layer of the dump and thereby impede percolation. As the water passes through the packed ore the viable thiobacilli decrease in number, an observation which could be taken as inconsistent with their proposed activity on sulphide ores. The massive dimensions of the leaching system, however, are likely to impose absorption and filtering out of bacteria and produce stratification and localized accumulations of organisms. In turn, localized heating, up to $80^{\circ}$, would have a twofold effect-a decrease in the bacterial population, and a possible enhancement of chemical oxidation at the expense of microbiological activity.

The exact contributions which bacteria make to copper leaching are not known fully, but the efficacy of their activity is determined by a complex interaction of physical, chemical and biological factors. Ehrlich and Fox (Biotech. Bioeng., IX, 471; 1967) ascribe three major functions to the thiobacilli: (1) direct oxidation of the sulphide ores; (2) regeneration of ferric iron which becomes involved in chemical oxidation of the ores; and (3) depressing the release of iron from the ores. Although it is possible only to assess the combined contributions made by thiobacilli at present, Ehrlich and Fox have studied parameters such as the nature of the gangue and mineralogy of the ore, particle size, the availability of oxygen and carbon dioxide, the $p \mathbf{H}, \mathbf{E}_{\mathrm{h}}$ and temperature, and the nutritional status of the system. This last point may affect the growth of heterotrophic micro-organisms, and either promote or inhibit the extraction process, by, for example, acid and sulphide production respectively. Unfortunately the scaling up of laboratory findings to field situations has had only limited success so far, and it is clear that more thorough evaluation of the in situ process along the lines of Beck's survey is a very necessary adjunct to simulated experiments in the laboratory.

\section{The Wake of the Moon}

\section{from our Magnetosphere Correspondent}

IN July 1967, the National Aeronautics and Space Administration in the United States succeeded in injecting the satellite Explorer 35 of the IMP 